\title{
Hongos asociados a la pudrición de la corona en frutos de banano orgánico (Musa spp. L.) en Piura, Perú
}

\section{Fungi associated with fruit crown rot in organic banana (Musa spp. L.) in Piura, Peru}

\section{Fungos associados à podridão-da-coroa em frutos de banana orgânica (Musa spp. L.) em Piura, Peru}

\author{
René Aguilar Anccota', Raquel Briggit García Raymundo², \\ José Antonio Dulanto Bejarano ${ }^{3}$ \& Edgar Abraham Maldonado Duque ${ }^{4}$

\begin{abstract}
${ }^{1}$ Ingeniero Agrónomo, Magister en Fitopatología. ${ }^{2}$ Ingeniera Agrónoma, estudiante de maestría en
${ }^{4}$ Ingeniero Agrónomo, estudiante del programa de maestría en Ciencias Agronómicas en la Universidad Particular Antenor Orrego ${ }^{1,4}$ Profesor Asociado, Departamento de Sanidad Vegetal, Universidad Nacional de Piura, Perú. ${ }^{2}$ Escuela de Postgrado, Universidad Nacional Agraria La Molina. Lima, Perú. ${ }^{3}$ Innovación y Desarrollo,

Profesionales Asociados S.A.C- Representante TASTE-PERU. Convenio de Investigación Fundación TASTE- Universidad Nacional de Piura, Perú.
\end{abstract} \\ la Universidad Nacional Agraria La Molina. ${ }^{3}$ Ingeniero Agrónomo, Magister en Producción Agrícola. \\ 1'raanccota@hotmail.com, ${ }^{2}$ briggit27@hotmail.com, \\ 3idulantob@master-srl.com, ${ }^{4}$ abrahammaldonado@hotmail.com
}

\section{Resumen}

El departamento de Piura es la principal zona productora de banano orgánico para la exportación en Perú, participando con el $87 \%$ del volumen de las exportaciones. En esta zona una de las enfermedades más importantes en postcosecha es la pudrición de la corona del fruto, las pérdidas económicas por esta enfermedad se han estimado entre el 25 y el $30 \%$ de la fruta exportada. El objetivo del presente estudio fue identificar los agentes causales asociados a esta enfermedad. Se evaluaron muestras de frutos de las zonas de Querecotillo, Salitral y Mallares empacados en cámaras de refrigeración, posteriormente se seleccionaron muestras con síntomas de pudrición de la corona, realizándose siembras de tejido enfermo en medio PDA+estreptomicina. Se aislaron e identificaron a
Thielaviopsis paradoxa, Lasiodiplodia theobromae, Colletotrichum musae y Fusarium verticillioides. Para demostrar la patogenecidad de las especies aisladas, se realizaron inoculaciones sobre la zona de la corona en frutos sanos de banano. Todos estos hongos causaron síntomas de infección en diferentes proporciones, concluyéndose que la pudrición de la corona es una enfermedad de etiología compleja.

Palabras clave: banano, pudrición de la corona, agentes causales, patogenicidad, etiología compleja.

\section{Abstract}

The department of Piura is the principal banana-producing zone in Peru, sharing $87 \%$ of exportations. 
In this zone, one of the most important postharvest diseases is crown rot. The economic loses attributed to this disease are estimated to be between 25 and $30 \%$ of organic bananas exported. The objective of this study was to identify the causal agents associated with this disease. Samples taken refrigerated fruit from the areas of Querecotillo, Salitral and Mallares were taken and selected after the fact. Thielaviopsis paradoxa, Lasiodiplodia theobromae, Colletotrichum musae and Fusarium verticilloides. In order to demonstrate the pathogenicity of the isolated species, inoculations were given in the area of the crown of the fruit on healthy bananas. These fungi caused symptoms of infection in different proportions, concluding that crown rot is a disease with a complex etiology.

Key-words: banana, crown rot, causal agents, pathogenicity, complex etiology.

\section{Resumo}

O departamento de Piura é a principal região produtora de banana orgânica para exportação no Peru, participando com $87 \%$ do volume de exportações. Nesta área, uma das mais importantes doenças pós-colheita é a podridão-da-coroa do fruto, as perdas econômicas desta doença são estimadas entre 25 e $30 \%$ dos frutos exportados. O objetivo deste estudo foi identificar os agentes causadores associados a esta doença. Amostras de frutas foram avaliadas nas áreas de Querecotillo, Salitral e Mallares e embaladas em câmaras de refrigeração, posteriormente foram selecionadas amostras com sintomas de podridão-dacoroa, realizando plantios do tecido doentes em meio PDA+estreptomicina. Foram isolados e identificados Thielaviopsis paradoxa, Lasiodiplodia theobromae, Colletotrichum musae e Fusarium verticillioides. Para demonstrar a patogenicidade das espécies isoladas, foram realizadas inoculações na coroa de frutos de bananas saudáveis. Todos estes fungos causaram sintomas da infecção em diferentes proporções, concluindo-se que a podridão-da-coroa é uma doença de etiologia complexa.

Palavras-chave: banana, podridão-da-coroa, agentes causais, patogenicidade, etiologia complexa.

\section{Introducción}

El banano es un cultivo que se desarrolla en la zona del valle del Chira, provincia de Sullana ubicada al norte del Perú, este cultivo beneficia a un gran número de pequeños productores que se insertan en la dinámica comercial de la exportación de banano orgánico y comercio justo, con una importancia económica significativa debido a la alta rentabilidad, así como por la gran demanda en el mercado internacional.

La región de Piura viene exportando frutos de banano orgánico desde el año 2002 y uno de los problemas más severos que se presentan durante la postcosecha es la enfermedad conocida como pudrición de la corona. Durante los meses de verano e invierno del año 2008 las pérdidas económicas ocasionadas por esta enfermedad fluctuaron entre 20 y $25 \%$ de la producción total (Dulanto, comunicación personal).
La pudrición de la corona se presenta en todo el mundo durante la fase de postcosecha en frutos de bananos y plátanos y se asocia a patógenos como: Fusarium spp.; Acremonium spp.; VerticiIlium sp.; Colletotrichum musae y Curvularia sp. (Ploetz, et al. 1994).

La enfermedad presenta un serio problema para la exportación ya que deteriora la calidad de la fruta cosechada y limita su aceptación por parte del consumidor. Con base en esta problemática el presente trabajo tuvo como objetivos: aislar e identificar los hongos fitopatógenos asociados a la pudrición de la corona y demostrar su patogenicidad de acuerdo a los postulados de Koch. 


\section{Metodología}

\section{Selección de muestras}

Se colectaron racimos de 8 plantas de banano recién cortadas, con un periodo de parición de cosecha de doce semanas, después del enfunde, de las zonas de Querecotillo, Salitral y Mallares, se llevaron a tinas de proceso que contienen alumbre, cloro y se procedió al desmane y al closteo con un tiempo de desleche de 15 minutos; posteriormente se realizó un empaque de manos de manera individual en bolsas de plástico (Consumer) tipo "cluster bag" y se empacaron en cajas, colocando la fruta en una cámara de refrigeración a una temperatura entre 13 a $15{ }^{\circ} \mathrm{C}$ por 10 días, para simular las condiciones del transporte marítimo. Posteriormente se seleccionaron las muestras con síntomas de pudrición en la zona de la corona, pedicelo y pulpa de la fruta; para los análisis de laboratorio respectivo.

\section{Aislamiento y purificación}

Las muestras con síntomas de pudrición se llevaron al laboratorio de Fitopatología de la Universidad Nacional de Piura para ser analizadas. Con un bisturí estéril se cortaron pequeños trozos de la zona de avance de la pudrición de 0,5 x 0,5 cm de tejido, estos se desinfectaron en una solución de hipoclorito de sodio al $1 \%$ durante $1 \mathrm{~min}$, luego se enjuagaron por dos veces con agua destilada (French y Hebert, 1980), y se dejaron secar por 10 min., posteriormente, una vez secas, los trozos se colocaron en placas Petri con medio Papa Dextrosa Agar (PDA) enmendado con estreptomicina $50 \mathrm{mg}$ y se incubaron a $28 \stackrel{\circ}{\circ} \mathrm{C}$ en oscuridad durante 5 días.

\section{Identificación de hongos}

Se prepararon montajes de cada uno de los aislamientos obtenidos y se hicieron observaciones utilizando un microscopio compuesto para analizar las características morfológicas del patógeno. La identificación de los hongos se realizó a través de las claves propuestas por Ellis (1976) y Sutton (1980).

\section{Ensayos de patogenicidad}

Se tomaron coronas sanas y sobre el área de corte se realizaron dos tipos de inoculación: primero, se colocó un disco de agar $(0,8 \mathrm{~cm}$ de diámetro) con micelio de los patógenos en estudio. En segundo lugar se asperjó una suspensión de conidias de los hongos a una concentración de $1 \mathrm{x}$ 10 conidias $/ \mathrm{ml}$, adicionalmente se asperjó sobre la superficie de la cáscara con la misma concentración de conidias de los hongos en estudio. En el tratamiento testigo se asperjó solamente agua estéril sobre la corona.

Todos los frutos inoculados se incubaron en cámara húmeda a temperatura ambiente por un periodo de 7 a 10 días.

\section{Resultados}

\section{Aislamientos}

Se obtuvieron cuatro aislamientos, se codificaron como: $A B-1, A B-2, A B-3$ y $A B-4$.

\section{Sintomatología e identificación}

El tipo de síntoma, coloración de la colonia y estructuras microscópicas se observan en las tablas 1,2 y 3

El aislamiento $A B$-1, se aisló de síntomas de pudrición blanda y oscura en la zona de la corona, pedicelo y pulpa, que comúnmente presentó un olor a fruta fermentada. Según Ploetz, et . al 1994, es debido al acetato de etilo y alcohol de etilo que produce el hongo. El aislamiento tuvo un crecimiento rápido y superficial en medio de cultivo PDA, las conidias fueron unicelulares pigmentadas de forma elipsoidal a ovoide con pared lisa, las conidias se 
formaron en cadena y promediaron de 7-14 $\mu \mathrm{m}$ de largo x 3-6 $\mu \mathrm{m}$ de ancho. Se encontraron fialides de color gris, las cuales tuvieron una longitud de $200 \mu \mathrm{m}$. Las características de los aislamientos se ajustan a las descritas para Thielaviopsisi paradoxa (de Seynes) Hohnel, (Figura 1. A y b) según Ellis (1971).

Un segundo aislado, nombrado $A B-2$, se aisló de una pudrición blanda en la zona de la corona, pedicelo y pulpa; desarrolló colonia algodonosa de crecimiento rápido de coloración gris oscuro. A los 5 días de siembra se observó la formación de picnidios, estos tuvieron forma redondeada de $200 \mu \mathrm{m}$ de diámetro; la pared del picnidio estuvo formada por varias capas de células de color gris oscuro. Al interior del picnidio se observaron células conidiógenas hialinas entremezcladas con parafisas hialinas y filamentosas. Las conidias al inicio fueron unicelulares de pared gruesa, hialinas de forma ovalada a elipsoide, y al madurar presentaron una septa en la parte media y estrías longitudinales sobre la superficie, estas tuvieron un tamaño promedio $23 \times 12 \mu \mathrm{m}$. Según la clave propuesta por Sutton (1980), se identificó como
Lasiodiplodia theobromae (Pat.) Griffon \& Maubl. (syn. Botriodiplodia theobromae) (Figura 1. c y d).

Un tercer aislado, $A B-3$, se aisló de manchas necróticas de la superficie de la cáscara y pudrición en la corona y pedicelo. El aislado tuvo una colonia algodonosa de color crema grisácea de crecimiento rápido, sobre la superficie del medio de cultivo se observaron masas conidiales de color salmón anaranjado, con la clave propuesta por Sutton (1980). El aislamiento se identificó como Colletotrichum musae (Fig 2. a y b).

Un cuarto aislado, denominado $A B-4$, se aisló de pudriciones secas en la corona y pedicelo, en medio PDA desarrolló una colonia algodonosa, de crecimiento lento, y de una pigmentación rojo violáceo. Las microconidias fueron unicelulares, hialinas de forma ovalada a alargadas cuyo tamaño fue de $6 \times 3$ $\mu$, las macroconidias fueron escasas, hialinas, finas, multicelulares con un promedio fue de $24 \times 5 \mu$, y no se observó la formación de clamidosporas, estas características coincidieron para la especie Fusarium verticillioides según la clave de Toussoun y Nelson, (1968) (Fig 2. c y d)

Tabla 1. Características morfológicas y fisiológicas de cuatro especies fúngicas aisladas de frutos de banano orgánico, procedentes del Valle del Chira, Piura 2010.

\begin{tabular}{l|cccc}
\hline \multirow{2}{*}{$\begin{array}{c}\text { Características } \\
\text { de la colonia }\end{array}$} & \multicolumn{4}{c}{ Aislados } \\
\cline { 2 - 5 } & $\begin{array}{c}\text { Thielaviopsis } \\
\text { paradoxa }\end{array}$ & $\begin{array}{c}\text { Lasiodiplodia } \\
\text { theobromae }\end{array}$ & $\begin{array}{c}\text { Colletotrichum } \\
\text { musae }\end{array}$ & $\begin{array}{c}\text { Fusarium } \\
\text { verticillioides }\end{array}$ \\
\hline Color & 0 & $\mathrm{~g}, \mathrm{o}$ & $\mathrm{g}, \mathrm{c}, \mathrm{n}$ & $\mathrm{g}, \mathrm{v}$ \\
Textura & $\mathrm{s}$ & $\mathrm{a}$ & $\mathrm{a}$ & $\mathrm{a}$ \\
Borde & $\mathrm{e}$ & $\mathrm{e}$ & $\mathrm{e}$ & $\mathrm{e}$ \\
Pigmentación en medio & - & $\mathrm{o}$ & - & $\mathrm{v}$ \\
\hline Velocidad de crecimiento & $4.8 \mathrm{~cm} / \mathrm{día}$ & $7.38 \mathrm{~cm} / \mathrm{día}$ & $1.2 \mathrm{~cm} / \mathrm{día}$ & $1.6 \mathrm{~cm} / \mathrm{día}$ \\
\hline
\end{tabular}

Color: $\mathrm{o}=$ oscura, $\mathrm{c}=$ cremoso, $\mathrm{g}=$ gris $\mathrm{v}=$ violáceo $\mathrm{n}=$ naranja Textura: $\mathrm{a}=$ algodonoso, $\mathrm{s}=$ superficial Borde: $\mathrm{e}=$ entero Pigmentación: $\mathrm{v}$ = violácea 
Tabla 2. Tipo y características microscópicas de las estructuras reproductivas, de los cuatro aislados de hongos obtenidos de frutos de banano procedente del valle del Chira, Piura - 2010.

\begin{tabular}{|c|c|c|c|c|}
\hline \multirow[b]{2}{*}{ Características } & \multicolumn{4}{|c|}{ Aislados de hongos } \\
\hline & $\begin{array}{c}\text { Thielaviopsis } \\
\text { paradoxa }\end{array}$ & $\begin{array}{l}\text { Lasiodiplodia } \\
\text { theobromae }\end{array}$ & $\begin{array}{c}\text { Colletotrichum } \\
\text { musae }\end{array}$ & $\begin{array}{l}\text { Fusarium } \\
\text { verticillioides }\end{array}$ \\
\hline $\begin{array}{l}\text { Cuerpo fructificante } \\
\text { Acérvulo } \\
\text { Picnidio (dim.) }\end{array}$ & $\begin{array}{l}\mathrm{nf} \\
\mathrm{nf}\end{array}$ & $350^{n f}$ & $\begin{array}{l}\text { sf } \\
\text { nf }\end{array}$ & $\begin{array}{l}\mathrm{nf} \\
\mathrm{nf}\end{array}$ \\
\hline $\begin{array}{l}\text { Conidióforo } \\
\text { Tipo } \\
\text { Longitud }(\mu \mathrm{m}) \\
\text { Diámetro }(\mu \mathrm{m})\end{array}$ & $\begin{array}{l}s f \\
50 \\
4-6\end{array}$ & $\begin{array}{c}\text { corto y simple } \\
5-15 \\
2.5-3\end{array}$ & $\begin{array}{c}\text { corto y simple } \\
-- \\
--\end{array}$ & $\begin{array}{c}\text { corto y simple } \\
20-30 \\
2-3\end{array}$ \\
\hline $\begin{array}{l}\text { Conidia } \\
\text { Largo }(\mu \mathrm{m}) \\
\text { Ancho }(\mu \mathrm{m})\end{array}$ & $\begin{array}{l}10-25 \\
8-16\end{array}$ & $\begin{array}{l}20-30 \\
10-15\end{array}$ & $\begin{array}{c}12-17 \\
4.5-5.5\end{array}$ & $\begin{array}{l}\text { M 20-24 x 3.5-4 } \\
\text { m 5-12 x 1.5-2.5 }\end{array}$ \\
\hline $\begin{array}{l}\text { Otras estructuras } \\
\text { Parafisas }(\mu \mathrm{m}) \\
\text { Apresorio }(\mu \mathrm{m}) \\
\text { Fialides }(\mu \mathrm{m})\end{array}$ & $\begin{array}{c}\mathrm{nf} \\
\mathrm{nf} \\
200\end{array}$ & $\begin{array}{l}50 \\
\mathrm{nf} \\
\mathrm{nf}\end{array}$ & $\underset{\substack{n f \\
n f}}{11 \times 10.25}$ & $\begin{array}{c}n f \\
n f \\
20-24 \times 3.5-4\end{array}$ \\
\hline
\end{tabular}

$\mathrm{M}=$ macroconidias $\quad \mathrm{m}=$ microconidias $\mathrm{sf}=\mathrm{si}$ forma $\mathrm{nf}=\mathrm{no}$ forma

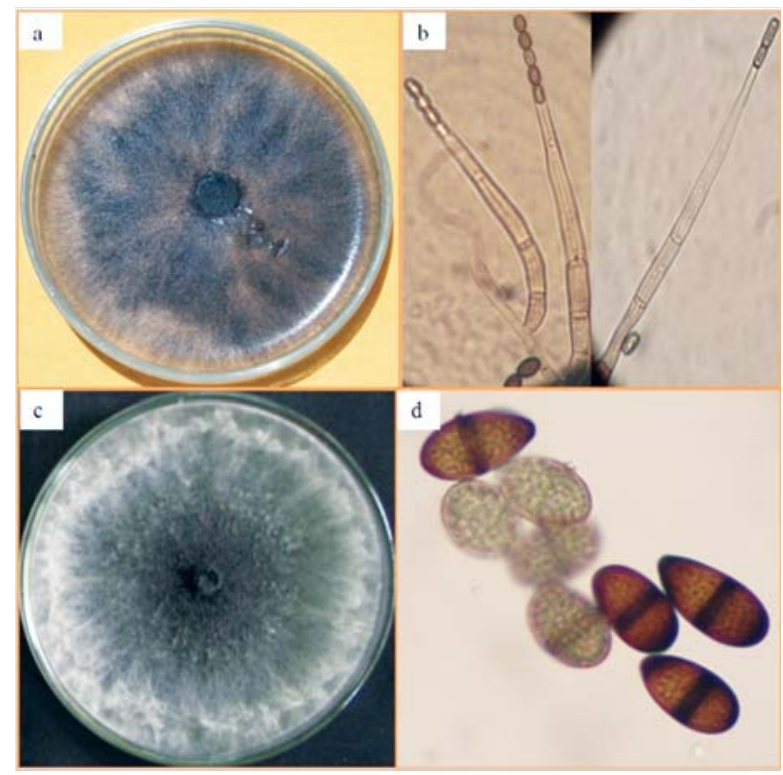

Figura 1. Hongo Thielaviopsis paradoxa a) Colonia oscura de crecimiento superficial en medio PDA

b) Conidias unicelulares oscuras en cadena de forma elipsoidal a ovoide, pared lisa. 7-14 $\mu \mathrm{m} \times 3-6$ $\mu \mathrm{m}$; fíalide $200 \mu \mathrm{m}$ (Ellis, 1971). Hongo Lasiodiplodia theobromae b) colonia algodonosa grisácea de crecimiento rápido en medio PDA c) conidias inmaduras unicelulares y bicelulares (maduras) 20-30 $\mu \mathrm{m} \times 10-15 \mu \mathrm{m}$ (Sutton, 1980).

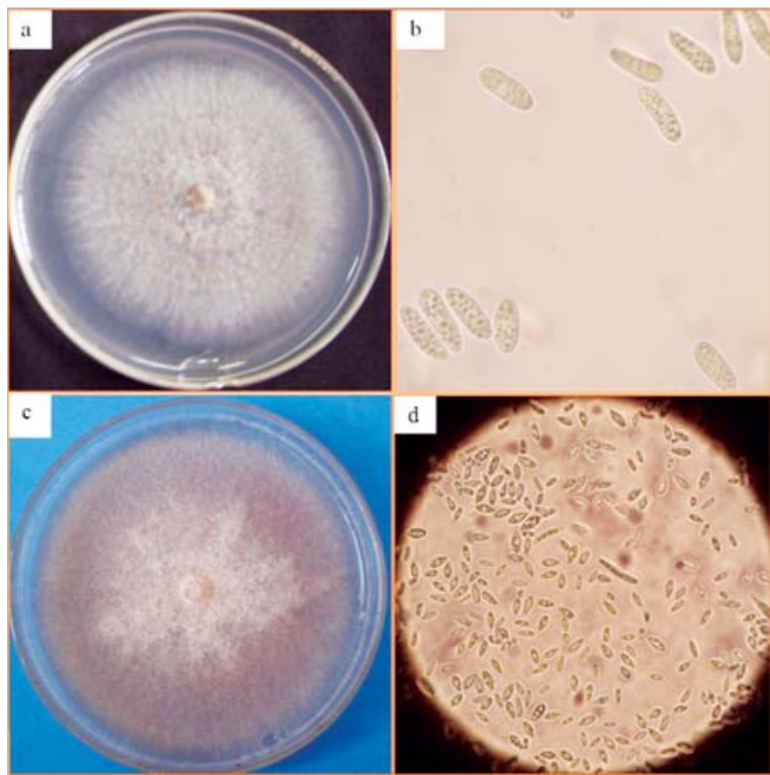

Figura 2. Hongo Colletotrichum musae a) colonia de crecimiento algodonoso de coloración gris anaranjado en medio de cultivo PDA b) conidias unicelulares de forma cilíndrica y alargada de 12-17 $\mu \mathrm{m} \times$ 4.5-5.5 $\mu \mathrm{m}$

(Sutton, 1980). Hongo Fusarium verticillioides c) Colonia de crecimiento algodonoso con pigmentación rojo violáceo d) abundante formación de microconidias unicelulares de forma ovoide y alargado, poca formación de macroconidias y clamidosporas ausentes (Toussoun y Nelson, 1968). 
Ensayo de patogenicidad: a los 6 días después de la inoculación, los cuatro hongos aislados e inoculados reprodujeron síntomas de pudrición en la corona y únicamente el hongo Colletorichum musae; reprodujo manchas necróticas en la superficie de la fruta. Según los autores Prusky, 1996 y Casarrubias et al. (2002) indican que el hongo $C$. musae forma un tubo germinativo que penetra de manera directa la cutícula del fruto inmaduro donde permanece latente, pero se reactiva la invasión durante la maduración del fruto.
De acuerdo a Lapeyre de Bellaire y Mourichon (1997), C. musae es el agente causal de la antracnosis de los bananos y plátanos, C. musae produce enzimas pectinolíticas y celuloliticas que degradan los componentes de la pared celular del hospedante (Rodríguez et al., 2009).

Según Ploetz, et al., 2003, indica que los hongos que causan pudrición de la corona se encuentran en campos de banano colonizando hojas muertas e inflorescencias, las conidias son dispersadas por el viento, agua de riego, insectos y aves.

Tabla 3. Sintomatología en frutos de Banano afectados por cuatro especies fúngicas procedentes del valle del Chira, Piura 2010.

\begin{tabular}{l|cccc}
\hline \multirow{2}{*}{ Aislamientos } & \multicolumn{4}{c}{ Partes del fruto afectadas } \\
\cline { 2 - 4 } & Corona & Pedicelo & Pulpa & Epidermis \\
\hline Thielaviopsis paradoxa & $\mathrm{Pb}, \mathrm{F}$ & $\mathrm{Pb}, \mathrm{F}$ & $\mathrm{Pb}, \mathrm{F}$ & - \\
Lasiodiplodia theobromae & $\mathrm{Pb}, \mathrm{F}$ & $\mathrm{Pb}, \mathrm{F}$ & $\mathrm{Pb}, \mathrm{F}$ & - \\
Colletotrichum musae & $\mathrm{Pb}, \mathrm{f}$ & $\mathrm{Pb}, \mathrm{f}$ & - & $\mathrm{Mn}, \mathrm{f}$ \\
Fusarium verticillioides & $\mathrm{Ps}, \mathrm{f}$ & $\mathrm{Ps}, \mathrm{f}$ & - & - \\
\hline
\end{tabular}

Pb: Pudrición blanda. Mn: Manchas necróticas. Ps: Pudrición seca. F: Fétida. f: fragante.

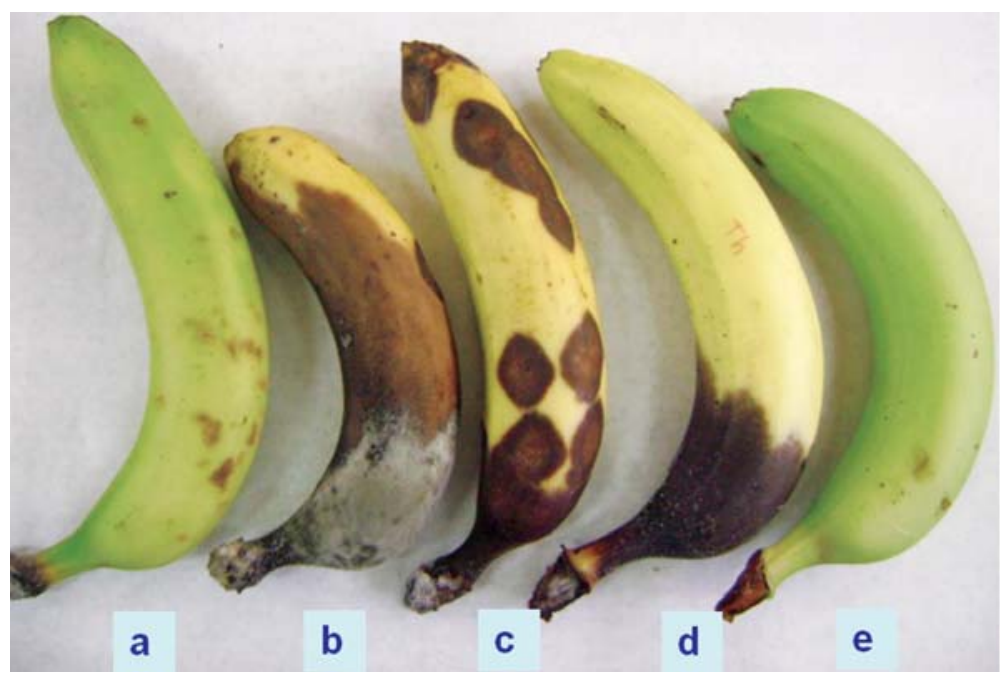

Figura 5. Ensayo de patogenicidad, con inoculación de los siguientes hongos: a) Fusarium verticillioides b) Lasiodiplodia theobromae c) Colletotrichum musae d) Thielaviopsis paradoxa e) testigo sin inoculación. 


\section{Discusión y conclusiones}

La sintomatología de pudrición de la corona observada en frutos de banano fue variable mostrando pudrición blanda, pudrición seca, con una coloración marrón a negro afectando la zona de corona y pedicelo, algunos con olor a fruta fermentada y vítrea según Ploetz et al. (1994) reportan que es debido al acetato de etilo que produce el hongo T. paradoxa. En algunos frutos aparecieron áreas necróticas de forma ovalada que abarcaron amplios sectores de la superficie del fruto, apreciándose la formación de acérvulos con masas conidiales de color salmón anaranjado, esta sintomatología coincide con la enfermedad causada por C. musae descrito por Snowdon (1990). Según Prusky (1996) sostiene que C. musae forma un tubo germinativo que penetra de manera directa la cutícula del fruto inmaduro, donde permanece latente, reactivándose la invasión durante la maduración del fruto. Al reactivar su actividad durante la maduración, la infección causa la formación de manchas típicas de color marrón sobre las frutas maduras. También puede desarrollarse y llegar a pudriciones destructivas de los dedos de las frutas verdes durante el almacenamiento en frío de 12 a $14 \stackrel{\circ}{\circ}$, las manchas en las frutas al principio son aguadas, usualmente de forma irregular y amarillentas, se agrandan y pueden adquirir la forma de lente o de huso y se tornan de color marrón oscuro o negro con un margen amarillento aguado. Las masas de esporas de color naranja se desarrollan en el centro de las manchas en condiciones húmedas. La enfermedad prospera sobre la cáscara lesionada y se agrava debido a las rozaduras y heridas provocadas durante la manipulación en post-cosecha. El almacenamiento durante largos periodos y las fluctuaciones de altas temperaturas de almacenamiento favorecen el desarrollo de la antracnosis (Meredith, 1971; Ploetz et al. 1994; Snowdon, 1990).

En cuanto a los síntomas producidos por $L$. theobromae, se apreció pudrición blanda en la zona de la corona, pedicelo y pulpa presentándose una colonia algodonosa de coloración gris oscuro, con abundante formación de picnidios inmersos en el tejido infectado. En las condiciones de Piura se ha reportado a este patógeno afectando mango y palto (Rodriguez-Gálvez, 2010). Asimismo, la sintomatología producida por $F$. verticillioides se manifestó con una pudrición seca en la corona y pedicelo. Cabe destacar que el daño producido por este hongo no fue tan severo como los daños producidos por T. paradoxa y $L$. theobromae. Resulta importante destacar que este complejo de hongos de pudrición de corona se caracteriza porque se presenta durante todo el año en Piura y en diferentes épocas inciden unos más que otros; algunos hongos como Thielaviopsis paradoxa se manifiestan durante los meses de mayo a octubre cuando baja la temperatura, mientras que CoIletotrichum musae, Lasiodiplodia theobromae y Fusarium verticillioides se presentan todo el año, con una mayor severidad durante los meses de noviembre a abril cuando se incrementa la temperatura en el valle, según comunicación personal de Dulanto quien realizó una investigación, evaluación y seguimiento durante dos años entre el 2007 y 2009, determinando los factores que predisponen la pudrición de corona en poscosecha a nivel comercial. La prueba de patogenicidad en frutos, permitió determinar que los agentes causales de la pudrición de la corona en frutos de banano fueron: Thielaviopsis paradoxa, Lasiodiplodia theobromae, Colletotrichum musae y Fusarium verticillioides. Los hongos aislados causaron síntomas de infección en diferentes proporciones, concluyéndose que la pudrición de la corona es una enfermedad de etiología compleja.

\section{Agradecimiento}

Los autores agradecen a Agrofair de Holanda representado por el Grupo Hualtaco S.A.C en Perú, a la Fundación TASTE de Holanda, a Master Profesionales Asociados S.A.C, a Copdeban 
-Dole Perú, por el apoyo financiero del presente trabajo de investigación en beneficio de los productores de banano orgánico del Valle del Chira, Piura-Perú.

\section{Literatura citada}

1. Casarrubias, C. Cárdenas, S.; Nieto, A. \& Gutiérrez, A. (2002). Histología de frutos de papaya (Carica papaya L.) infectados por Colletotrichum gloeosporioides Penz. Revista Mexicana de Fitopatología, 20: 83-93.

2. Ellis, M.B. (1971). More Dematiaceous Hyphomycetes. Surrey: International Mycological Institut Kew.

3. French, E. R. \& Herbert, T. (1980). Métodos de investigación Fitopatología. San José: Editorial IICA.

4. Lapeyre de Bellaire, L. \& Mourichon, X. (1997). The pattern of Fungal Contamination of the banana Bunch During its Development and Potential Influence on Incidence of Crown Rot and Anthracnose Diseases, Plant Pathology, 46 (4): 481-489, Inglaterra.

5. Meredith, D. S. (1971). Transport and storage diseases of bananas: Biology and control. Trop.Agric. (Trinidad) 48(1):35-50.

6. Ploetz, C., A. Zentmeyer, T., Nishijima, G., Rohrbach \& D. Ohr. (1994). Compendium of tropical Fruit diseases. St. Paul, MN.: APS Press.
7. Ploetz, R. C., Thomas, J. E., \& Slabaugh, W. (2003). Disease of banana and plantain. Pp. $73-134$ in: R. C. Ploetz, ed. Diseases of Tropical Fruit Crops. London: Cabi International.

8. Prusky, D. (1996). Pathogen quiensance in postharvest diseases. Annual Review Phytopathology 34: 413-434

9. Rodríguez L.E., González P.J. \& Mayek P.N. (2009). La infección de Colletotrichum gloeosporioides Penz. y Sacc. en Aguacatero (Persea americana Mill.): aspectos bioquímicos y genéticos. Revista Mexicana de Fitopatología. vol.27, 1: 53-63.

10. Rodríguez-Gálvez, (2010). Lasiodiplodia theobromae Fitopatógeno de Mango (Manguífera indica) y Palto (Persea americana) Departamento de Sanidad Vegetal. Facultad de Agronomía. Universidad Nacional de Piura.

11. Snowdon, A. L. (1990). A colour atlas of post-harvest diseases and disorders of fruits and vegetables Vol. 1: General introduction and fruits. London: Wolfe Scientific Publications.

12. Sutton, B. (1980). The Coelomycetes. Surrey: Commonwealth Mycological Institute, Kew.

13. Toussoun, T. A. \& Nelson, P. E.. (1968). A pictorial guide to the identification of Fusarium species according to the taxonomic system of Snyder and Hansen. Pennsylvania: The Pennsylvania State University Press, University Park. 